\title{
Gs $\alpha$ Is Involved in Sugar Perception in Drosophila melanogaster
}

\author{
Kohei Ueno, ${ }^{1}$ Soh Kohatsu, ${ }^{3}$ Catherine Clay, ${ }^{4}$ Michael Forte, ${ }^{4}$ Kunio Isono, ${ }^{3}$ and Yoshiaki Kidokoro ${ }^{1,2}$ \\ ${ }^{1}$ Department of Behavioral Sciences, Graduate School of Medicine, and ${ }^{2}$ Institute for Molecular and Cellular Regulation, Gunma University, Maebashi 371- \\ 8511, Japan, ${ }^{3}$ Graduate School of Information Sciences, Tohoku University, Sendai 980-8579, Japan, and ${ }^{4}$ Vollum Institute of Oregon Health and Science \\ University, Portland, Oregon 97239
}

In Drosophila melanogaster, gustatory receptor genes (Grs) encode G-protein-coupled receptors (GPCRs) in gustatory receptor neurons (GRNs) and some olfactory receptor neurons. One of the Grgenes, Gr5a, encodes a sugar receptor that is expressed in a subset of GRNs and has been most extensively studied both molecularly and physiologically, but the G-protein $\alpha$ subunit (G $\alpha$ ) that is coupled to this sugar receptor remains unknown. Here, we propose that $\mathrm{Gs}$ is the $\mathrm{G} \alpha$ that is responsible for Gr5a-mediated sugar-taste transduction, based on the following findings: First, immunoreactivities against Gs were detected in a subset of GRNs including all Gr5a-expressing neurons. Second, trehalose-intake is reduced in flies heterozygous for null mutations in $D G s \alpha$, a homolog of mammalian Gs, and trehalose-induced electrical activities in sugar-sensitive GRNs were depressed in those flies. Furthermore, expression of wild-type $D G s \alpha$ in sugar-sensitive GRNs in heterozygotic $D G s \alpha$ mutant flies rescued those impairments. Third, expression of double-stranded RNA for DGs $\alpha$ in sugarsensitive GRNs depressed both behavioral and electrophysiological responses to trehalose. Together, these findings indicate that $D G s \alpha$ is involved in trehalose perception. We suggest that sugar-taste signals are processed through the Gs $\alpha$-mediating signal transduction pathway in sugar-sensitive GRNs in Drosophila.

Key words: gustatory receptor; Gs; Drosophila melanogaster; sweet taste; trehalose; cAMP transduction pathway

\section{Introduction}

Sugar is a major source of energy for animals, and its taste is appealing, but the transduction pathway by which animals detect sugar in their environment and then process sugar-taste information into neuronal signals remains undetermined. In Drosophila melanogaster, gustatory receptor neurons (GRNs) express $\sim 60$ gustatory receptor genes (Grs), which are members of the G-protein-coupled receptor (GPCR) family (Clyne et al., 2000; Dunipace et al., 2001; Scott et al., 2001; Robertson et al., 2003). The natural ligands recognized by the Grs are mostly unknown, except one that has been identified, namely trehalose ( $\alpha$-Dglucopyranosyl- $\alpha$-D-glucopyranoside), the receptor of which is encoded by Gr5a (Dahanukar et al., 2001; Ueno et al., 2001; Chyb et al., 2003). Gr5a is activated by trehalose and coupled to the G-protein $\alpha$ subunit $(\mathrm{G} \alpha)$, which potentially routes the signal to several distinct transduction pathways (Neves et al., 2002; Wong, 2003). In this study, we wished to identify which $\mathrm{G} \alpha$ is coupled to the sugar-taste transduction pathway.

In the Drosophila genome, 11 genes encode $\mathrm{G} \alpha$ (Ishimoto et al., 2005), and one of them, $D G s \alpha$, is a homolog of mammalian

Received 0ct. 16, 2005; revised April 14, 2006; accepted April 15, 2006.

This work was supported by grants to K.U. and Y.K. from a Centers of Excellence grant to Gunma University and a grant-in-aid from the Ministry of Education, Culture, Sports, Science, and Technology of Japan. We thank H. Amrein for the gift of Gr66a-GAL4 transgenic flies and W. L. Pak for the gift of norpA ${ }^{\mathrm{P} 24}$ mutants. We also thank H. Kuromi, T. Sakai, and A. Honda for critical comments and M. Wakai for her assistance in rearing the flies.

Correspondence should be addressed to Kohei Ueno, Department of Behavioral Sciences, Graduate School of Medicine, Gunma University, Maebashi 371-8511, Japan. E-mail: kueno@med.gunma-u.ac.jp.

DOI:10.1523/JNEUROSCI.0857-06.2006

Copyright $\odot 2006$ Society for Neuroscience $\quad$ 0270-6474/06/266143-10\$15.00/0
Gs (Quan et al., 1989). The primary function of the Gs family is to elevate the concentration of cAMP via adenylyl cyclase (AC), and in vertebrates, this transduction pathway is involved in a variety of cellular functions (Tesmer and Sprang, 1998; Hurley, 1999; Simonds, 1999). Electrophysiological and biochemical studies have shown that CAMP is involved in sugar taste in vertebrates, although it is still unclear which isoform of Gs is coupled to the sugar receptor (Avenet and Lindemann, 1987; Striem et al., 1989; Naim et al., 1991). Gs is also involved in other sensory functions, for example, olfactory signaling in the rat is mediated by Golf, an isoform of Gs, and Golf is expressed in olfactory receptor neurons, localized at cilia and coupled to type III AC (Jones and Reed, 1989; Menco et al., 1992).

The Drosophila homolog DGs $\alpha$ is likely to play an important role in various neuronal functions, because expression of a constitutively active form of $D G s \alpha$ in the mushroom bodies impairs the learning abilities of flies (Connolly et al., 1996). Furthermore, $D G s \alpha$ is required for the normal growth and function of synapses (Wolfgang et al., 2004), and synaptic transmission at the neuromuscular junction is compromised in $D G s \alpha$-null mutant embryos (Hou et al., 2003). Thus, DGs $\alpha$ might be the $\mathrm{G} \alpha$ that is coupled to Grs.

To determine whether the $\mathrm{G} \alpha$-mediating transduction pathway is involved in sugar-taste signaling in Drosophila, we first immunohistochemically demonstrated the localization of the DGs $\alpha$ protein in GRNs. Next, we showed that gustatory responses were depressed in heterozygous $D G s \alpha$-null mutant flies using behavioral and electrophysiological assays. These impaired 
phenotypes were rescued by expression of wild-type $D G s \alpha$ in GRNs in the mutants. We also found that reduced DGs $\alpha$ expression induced by the RNAi technique depressed the trehalose responses. Together, we conclude that DGs $\alpha$ is involved in sugar perception and suggest that Gs $\alpha$ mediates sugar-taste signaling in Drosophila.

\section{Materials and Methods}

Fly cultures. All flies were reared on standard cornmeal medium at $25 \pm$ $2{ }^{\circ} \mathrm{C}$, in $60 \%$ relative humidity and under a $12 \mathrm{~h}$ light/dark cycle. They were used for experiments on $2-5 \mathrm{~d}$ after eclosion.

Construction of a transgene Gr5a-GAL4. Gr5a-GAL4 was constructed by first generating a PCR product of $853 \mathrm{bp}$, corresponding to a sequence between that immediately upstream of the ATG first codon of Gr5a and the transcriptional starting site of an adjacent gene, CG3171, from Drosophila genomic DNA. This putative Gr5a upstream fragment and GAL4 sequence were subcloned into a $\mathrm{pP}\{\mathrm{CaSpeR}-4\}$ vector. Injecting the Gr5a-GAL4 constructs, $w \mathrm{Gr}_{5 a^{+}}$; Gr5a-GAL4 flies were generated.

Immunohistology. Labela were dissected from heads and fixed in $4 \%$ paraformaldehyde/PBS for $30 \mathrm{~min}$ at $4^{\circ} \mathrm{C}$. Primary antibodies used were rabbit anti-Gs $\alpha$ peptide antiserum (catalog \#sc-383; Santa Cruz Biotechnology, Santa Cruz, CA) at a 1:100 dilution and anti-GFP $\operatorname{IgG}_{2 \mathrm{a}}$ (catalog \#A11120; Invitrogen, Carlsbad, CA) at a 1:200 dilution. The Gs $\alpha$ peptide corresponds to the sequence of $18 \mathrm{C}$-terminal amino acid residues, which is a conserved sequence of the Gs $\alpha$ family (Wolfgang et al., 1990). Secondary antibodies were goat anti-rabbit IgG coupled to Alexa-568 (\#A21069; Invitrogen) at a 1:200 dilution and goat anti-mouse IgG coupled to Alexa-488 (\#A-11017; Invitrogen) at a 1:200 dilution.

DGs $\alpha$ mutants. Two strains of $D G s \alpha$-null mutants were used: $c n b w$ $D G s \alpha^{\mathrm{R} 19} / \mathrm{SM} 6$ and $c n$ bw DGs $\alpha^{\mathrm{R} 60} / \mathrm{SM} 6$. Because homozygous $D G s \alpha$ mutations are lethal (Wolfgang et al., 2001), we generated heterozygous male flies ( $c n b w D G s \alpha^{\mathrm{R} 19} /+$, cn $b w D G s \alpha^{\mathrm{R} 60} /+$ and $c n b w /+$ ) by crossing female flies of a wild-type strain (Canton-S) with male flies of either one of the $D G s \alpha$ strains or $c n b w$ and used $c n b w /+$ flies as a control for both strains of heterozygous $D G s \alpha$-null mutant flies (supplemental Table and Fig. 1, available at www.jneurosci.org as supplemental material).

Measurements of sugar intake. Procedures to measure the amount of trehalose intake have been described previously (Shimada et al., 1987). Briefly, after $9 \mathrm{~h}$ of starvation, $\sim 30$ flies were allowed to feed on sugar solutions containing $1 \%$ agar and a blue food dye $(0.125 \mathrm{mg} / \mathrm{ml}$ brilliant blue FCF) in the dark for $1 \mathrm{~h}$ on a 60 -well micro-test plate (Nunc, Roskilde, Denmark). After this feeding session, flies were killed at $-20^{\circ} \mathrm{C}$ and homogenized with $500 \mu \mathrm{l}$ of PBS/EtOH. After centrifugation at 15,000 $\mathrm{rpm}$ for $10 \mathrm{~min}$, absorbance of the supernatant was measured at $630 \mathrm{~nm}$ by a spectrophotometer (GE Healthcare Bio-Sciences, Piscataway, NJ). The absolute amount of intake was calculated from a calibration curve of the dye. Flies drink a certain amount of water regardless of sugar. To correct for this offset of intake, the mean amount of water intake was measured separately and subtracted from the mean amount of intake of sugar solutions for a given group of flies. In all experiments, the amount of water intake in each fly was not different among flies with various genetic backgrounds used in this study $(p>0.05)$.

Two-choice test with bitter solutions. Before the behavior test, flies were starved for $9 \mathrm{~h}$ in empty vials. Thirty to forty of those flies were introduced onto a 60-well micro-test plate and allowed to feed in the dark for $1 \mathrm{~h}$. The wells in a micro-test plate were alternately filled with bitter and control solutions that were colored with red and blue food dyes, respectively. All solutions contained $1 \%$ agar. The concentration of food dye was $0.5 \mathrm{mg} / \mathrm{ml}$ for amaranth and $0.25 \mathrm{mg} / \mathrm{ml}$ for brilliant blue FCF. Because the quinine and denatonium benzoate solutions were acidic, we neutralized them with HEPES buffer ( $10 \mathrm{~mm})$, pH 7.0. After feeding, flies were killed in a freezer and classified under a dissection microscope into four groups according to their abdominal color: blue $(\mathrm{Nb})$, red $(\mathrm{Nr})$, purple $(\mathrm{Np})$, and no staining $(\mathrm{Nn})$. The preference index (PI) of the control solution over a bitter solution was calculated as $[(\mathrm{Nb}-\mathrm{Nr}) /(\mathrm{Nb}$ $+\mathrm{Nr}+\mathrm{Np})] \times 100$. The percentage of $\mathrm{Nn}$ flies was smaller than $10 \%$ in all experiments. The PI close to 100 indicates that the flies avoid the bitter solution. All behavioral tests were performed at $25^{\circ} \mathrm{C}$ and in $60 \%$ relative humidity.

GAL4/upstream activator sequence analysis. For the experiment shown in Figures 3, 4, and 5, we generated $c n$ bw DGs $\alpha^{\mathrm{R} 19} / \mathrm{SM} 6$; upstream activator sequence (UAS)-DGs $\alpha$ and crossed it with $G r 5 a^{+}$; Gr5a-GAL4 or $\mathrm{Gr}_{5} \mathrm{a}^{+}$(Canton-S). The UAS-DGs $\alpha$ strain carried a wild-type DGs $\alpha$ cDNA sequence linked to UAS (Wolfgang et al., 2001). Thus, we obtained $G r 5 a^{+}$; $c n b w D G s \alpha^{\mathrm{R} 19} / G r 5 a-G A L 4 ;$ UAS-DGs $\alpha /+$ and $w G r 5 a^{+}$; cn bw $D G s \alpha^{\mathrm{R} 19} /+$; UAS-DGs $\alpha /+$.

We crossed $c n$ bw DGs $\alpha^{\mathrm{R} 19} / \mathrm{SM} 6$ and $\mathrm{Gr} 5 a^{+}$; Gr5a-GAL4 to generate $\mathrm{Gr} \mathrm{a}^{+}$; cn bw DGs $\alpha^{\mathrm{R} 19} / \mathrm{Gr} 5 a-\mathrm{GAL} 4 ;+/+$.

For the experiment shown in Figure 6, we generated Gr5a $a^{\Delta \mathrm{EP} 19} ; \mathrm{Gr} 5 a$ GAL4 and crossed it with $c n b w D G s \alpha^{\mathrm{R} 19} / \mathrm{SM} 6$; UAS-DGs $\alpha$ or $c n b w$ $D G s \alpha^{\mathrm{R} 19} / \mathrm{SM} 6$ to generate Gr5a $a^{\Delta \mathrm{EP} 19}$; cn bw DGs $\alpha^{\mathrm{R} 19} / G r 5 a-G A L 4$; UAS-DGs $\alpha /+$ and Gr5a ${ }^{\Delta \mathrm{EP} 19}$; $c n$ bw $D G s \alpha^{\mathrm{R} 19} / G r 5 a-G A L 4 ;+/+$. We crossed Gr5a $a^{\Delta \mathrm{EP} 19}$ with $c n$ bw DGs $\alpha^{\mathrm{R} 19} / \mathrm{SM} 6$; UAS-DGs $\alpha$ to generate $G r 5 a^{\Delta \mathrm{EP} 19} ; c n$ bw DGs $\alpha^{\mathrm{R} 19} /+$; UAS-DGs $\alpha /+$. (supplemental Table and Fig. 2, available at www.jneurosci.org as supplemental material).

Electrophysiological recording of taste responses from GRNs. The response of GRNs to various substances was recorded from the L-III, L-V, and L-VII chemosensilla in a labelum (Hiroi et al., 2002) by the tip recording method (Hodgson et al., 1955). A reference glass electrode, containing the Ephrussi-Beadle Ringer's solution ( $128 \mathrm{~mm} \mathrm{NaCl}, 4.7 \mathrm{~mm}$ $\mathrm{KCl}$ ), was inserted in the abdomen of an anesthetized male fly, and its tip was placed in the head. To prevent changes in the stimulant concentration by evaporation, the solution in the recording capillary tube was constantly flowed out from the tip by positive pressure. All stimulant solutions contained $7.5 \mathrm{~mm} \mathrm{KCl}$ as an electrolyte. Signals were filtered with a low-pass filter $(2.5 \mathrm{kHz})$, digitized by an $\mathrm{A} / \mathrm{D}$ converter, and stored on a computer (Molecular Devices, Union City, CA). Spontaneous sugar spikes and/or salt spikes responded to $7.5 \mathrm{~mm} \mathrm{KCl}$. However, the number of spontaneous sugar spikes was very low (for example, $0.28 / 200 \mathrm{~ms}$ in $\mathrm{cn}$ $b w /+)$, and no significant difference was found among flies with various genetic backgrounds used in this study $(p>0.05)$. They were subtracted from all sugar responses to trehalose solutions in each chemosensillum.

UAS-DGs $\alpha$ RNAi analysis. UAS-DGs $\alpha$ RNAi was constructed with double-stranded RNA representing nucleotides -42 to 1381 of the transcript encoding DGs $\alpha$ and cloned into a pUAST vector at the EcoRI and $K p n I$ sites. This RNAi construct suppresses the expression of two isoforms of $D G s \alpha$ (Quan et al., 1989; Quan and Forte, 1990). The Gr66aGAL4 fly was a gift from Dr. Hubert Amrein (Duke University, Durham, NC). We crossed Gr66a-GAL4 flies and $\mathrm{Gr}^{2} a^{+}$(Canton-S) flies and generated $\mathrm{Gr}_{5} \mathrm{a}^{+}$; Gr66a-GAL4. To express DGs $\alpha$ RNAi in Gr5a-GRNs, we crossed UAS-DGs $\alpha$ RNAi males with $\mathrm{Gr}_{5 a^{+}}$; Gr5a-GAL4 females to generate $\mathrm{Gr}_{5 a^{+}}$; Gr5a-GAL4/UAS-DGs $\alpha$ RNAi. To express $D G s \alpha$ RNAi in Gr66a neurons, we crossed UAS-DGs $\alpha$ RNAi males with $G r 5 a^{+}$; Gr66a-GAL4 females to generate $\mathrm{Gr}_{5} a^{+}$; Gr66a-GAL4/UAS-DGs $\alpha$ RNAi. (supplemental Table and Fig. 3, available at www.jneurosci.org as supplemental material).

Western blotting. To express DGs $\alpha$ RNAi in the larval CNS, we crossed UAS-DGs $\alpha$ RNAi with 1407-GAL4 flies and generated 1407-GAL4/UAS$D G s \alpha$ RNAi larvae. The 1407-GAL4 expresses UAS transgene in the larval CNS (Luo et al., 1994). The CNS from third-instar larvae were dissected in a saline (in mM: $130 \mathrm{NaCl}, 36$ sucrose, $5 \mathrm{KCl}, 5 \mathrm{HEPES}, 2 \mathrm{MgCl}_{2}$, and 0.5 EGTA, pH 7.2) containing protease inhibitors. Subsequently, the saline was removed and replaced with $1 \times$ SDS gel sample buffer. After brief homogenization, the CNS tissue was incubated at $95^{\circ} \mathrm{C}$ for $4 \mathrm{~min}$. The equivalent of one larval CNS was then loaded per lane, and proteins were separated by electrophoresis on a $10 \%$ polyacrylamide gel. Separated proteins were transferred to nitrocellulose, and resulting blots were probed with the rabbit anti-Gs $\alpha$ peptide antiserum used in immunohistochemical studies described above. Blots were then probed with horseradish peroxidase-conjugated anti-rabbit secondary antibodies, and labeled bands were detected by incubating with chemiluminescent substrates.

norpA mutant. The norpA ${ }^{\mathrm{P} 24}$ mutant was a gift from Dr. William L. Pak (Purdue University, West Lafayette, IN). Because Gr5a and norpA are located near one another on the $\mathrm{X}$ chromosome, we crossed $w c x$ $G r 5 a^{+}$and norpA $A^{\mathrm{P} 24}$ and generated $w$ norp $A^{\mathrm{P} 24} c x G r 5 a^{+}$flies. $c x$ is 

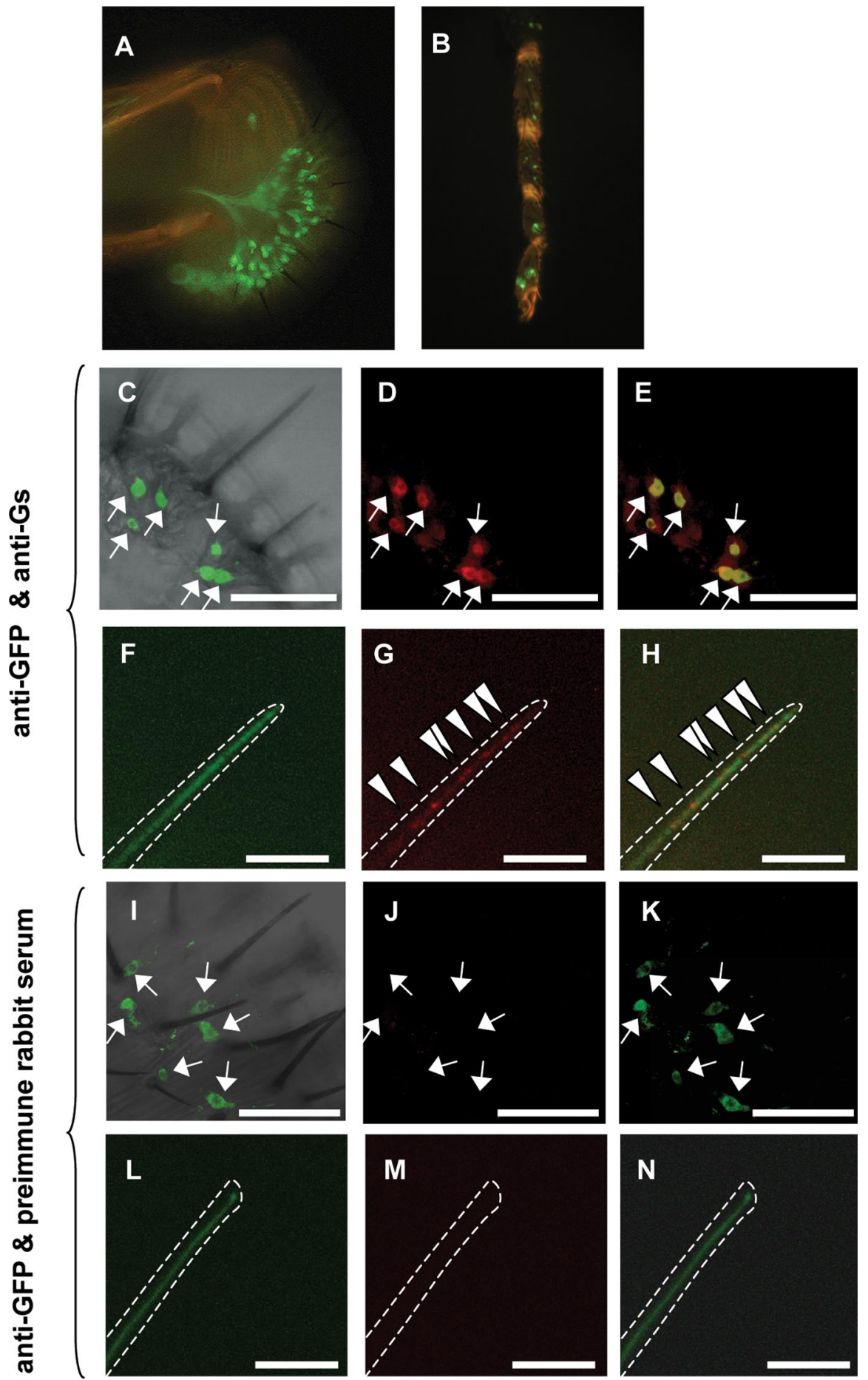

Figure 1. Expression of the DGs $\alpha$ protein in labela. GFP is expressed in a subset of GRNs in a labelum $(\boldsymbol{A})$ and tarsus $(\boldsymbol{B})$ but not in nongustatory tissues in Gr5a-GAL4/UAS-mCD8::GFP flies. C, I, Merged images of immunofluorescence stained with anti-GFP antibody (arrows) and a transmission light image of a labelum of a Gr5a-GAL4/UAS-mCD8::GFP fly. D, An image of immunofluorescence stained with an anti-Gs $\alpha$ antiserum (red) of the same labelum as in $\boldsymbol{C}$. $\boldsymbol{E}$, A merged image of immunofluorescence stained with the anti-GFP antibody (C) and with the anti-Gs $\alpha$ antiserum (D). The GRNs that reacted to the anti-GFP antibody (arrows) also reacted to the anti-Gs $\alpha$ antiserum, resulting in yellow, but some GRNs reacted only to the anti-Gs $\alpha$ antiserum (red). $\boldsymbol{F}, \boldsymbol{L}, \mathrm{A}$ high-magnification image of a chemosensillum. Anti-GFP fluorescence (green) was diffuse. In contrast, anti-Gs $\alpha$ fluorescence (red) was in clusters indicated by arrowheads $(\boldsymbol{G})$. $\boldsymbol{H}$, Merged images of $\boldsymbol{F}$ and $\boldsymbol{G}$. $\boldsymbol{J}, \boldsymbol{M}$, An image of immunofluorescence treated with preimmune rabbit serum of the same labelum as in $\boldsymbol{I}$ and the same chemosensillum as in $\boldsymbol{L}$. $\boldsymbol{K}$, A merged image of $\boldsymbol{I}$ and $\boldsymbol{J}$. $\boldsymbol{N}$, A merged image of $\boldsymbol{L}$ and $\boldsymbol{M}$. There was no red signal in the labelum or in the chemosensillum treated with the preimmune rabbit serum $(\boldsymbol{J}$ and $\boldsymbol{M})$. The white dotted line indicates the outline of a chemosensillum. Scale bars: $\boldsymbol{C}-\boldsymbol{E}, \boldsymbol{I}-\boldsymbol{K}$, $50 \mu \mathrm{m} ; \boldsymbol{F}-\boldsymbol{H}, \boldsymbol{L}-\mathbf{N}, 5 \mu \mathrm{m}$. located close to $G r 5 a$ and tightly linked to the $\mathrm{Gr} \mathrm{a}^{+}$allele (Ueno et al., 2001). Because norpA is essential for phototransduction (Bloomquist et al., 1988), we first recorded the electroretinogram in these flies and confirmed a lack of response to orange light (data not shown). In the behavioral and electrophysiological tests, we used $w c x \mathrm{Gr}_{5} \mathrm{a}^{+}$as a control.

Chemicals. Trehalose $(\mathrm{D}(+)$-trehalose dihydrate), sucrose, fructose $(\mathrm{d}(-)$-fructose), glucose $(\mathrm{D}(+)$-glucose), caffeine (caffeine anhydrous), quinine (quinine hydrochloride dihydrate), denatonium benzoate, and brilliant blue FCF were purchased from Wako Pure Chemical Industries (Osaka, Japan). Amaranth was purchased from Sigma-Aldrich Corporation (St. Louis, MO).

Statistical analysis. We used the Student's $t$ test for paired comparisons and the one-way ANOVA followed by the Sheffe's test for multiple comparisons. We also used the Steel-Dwass test to compare the numbers of impulses that were not normally distributed.

\section{Results}

$D G s \alpha$ is expressed in gustatory receptor neurons

If $D G s \alpha$ were required for taste signaling, we would expect the DGs $\alpha$ protein to be expressed in GRNs. As expected, we detected mRNA of $D G s \alpha$ in labela by reverse transcription-PCR analysis (data not shown). We then immunohistochemically examined the localization of the DGs $\alpha$ protein in GRNs using an antiserum against a Gs peptide. To this end, we first examined the distribution of GFP expressed in GRNs in transgenic flies carrying Gr5a-GAL4 and UAS-GFP under a fluorescence stereomicroscope and found that GFP was expressed specifically in a subset of GRNs in labela and tarsi (Fig. $1 A, B)$. This finding is in accord with previous reports (Chyb et al., 2003; Thorne et al., 2004; Wang et al., 2004).

Under a confocal microscope, a set of GFP-expressing GRNs was located near the proximal end of the chemosensillum (Fig. 1C,I, green cells, indicated by arrows). The immunofluorescence against Gs was observed in GFP-expressing GRNs ( $G r 5 a-G R N s$, indicated by arrows) as well as in nonexpressing GRNs (non-Gr5a GRNs) (Fig. 1D,E). All Gr5a-GRNs had anti-Gs immunoreactivities. We counted the numbers of Gr5a-GRNs and other GRNs that showed anti-Gs immunoreactivities in a labelum. They were $35 \pm 3$ and $77 \pm 6(n=5)$, respectively. The number of Gr5a-GRNs that we found is close to that in previous reports $[\sim 30$ in a labelum (Chyb et al., 2003) and $71 \pm 11$ in a palp that contains two labela (Thorne et al., 2004)]. From the morphological and electrophysiological experiments, the total 
number of GRNs in a labelum is estimated to be $\sim 150$ (Amrein and Thorne, 2005). Hence, our results indicate that approximately one-half of GRNs are expressing $D G s \alpha$, although the intensity of immunofluorescence against Gs in Gr5a-GRNs was higher than that in non-Gr5a GRNs (Fig. $1 D)$. In the chemosensillum, GRNs extended their dendrites (Fig. $1 F, L$ ), and anti-Gs fluorescence clusters were found at the dendrite, revealing punctuated localization of Gs (Fig. 1G,H, arrowheads). Immunoreactivities in GRNs were not detected in negative controls, in which preimmune rabbit serum was used (Fig. $1 J, K, M, N$ ). These results indicate that $D G s \alpha$ is expressed in GRNs, including all Gr5a-GRNs, in a labelum.

\section{Sugar intake is depressed in}

heterozygous DGs $\alpha$-null mutants

To examine the behavioral response to sugars in heterozygous $D G s \alpha$-null mutant flies, we measured the amount of intake of $20 \mathrm{~mm}$ trehalose, $5 \mathrm{~mm}$ sucrose, $20 \mathrm{~mm}$ fructose, and $20 \mathrm{~mm}$ glucose in $D G s \alpha^{\mathrm{R} 19} /+$ and in $c n b w /+$, a control. The amount of intake of each sugar solution in $D G s \alpha^{\mathrm{R} 19} /+$ flies was significantly lower than that in control flies (Fig. 2A) $(p<0.05)$.

It is known that a variety of gustatory receptors, Gr22b, Gr22c, Gr22e, Gr22f, $G r 28 b, G r 32 a, G r 59 b$, and Gr66a, are expressed in non-Gr5a GRNs and that those GRNs are required for bitter taste perception (Thorne et al., 2004; Wang et al., 2004). It is then possible that bitter-taste perception is also mediated by Gs $\alpha$ and that the $D G s \alpha$-expressing GRNs other than $G r 5 a$ (Fig. 1D,E) are bitter-sensitive GRNs. To test this possibility, we examined the behavioral response to bitter substances in $D G s \alpha^{\mathrm{R} 19} /+$ flies using the twochoice test. We tested three bitter substances, caffeine (at 1, 5, and $10 \mathrm{~mm}$ ), quinine (at $0.1,0.25,0.5,1$, and $2.5 \mathrm{~mm}$ ), and denatonium benzoate (at $0.025,0.1,0.25,0.5$, and $1 \mathrm{~mm}$ ). These bitter substances have been shown to induce the behavioral and electrophysiological bitter responses in Drosophila (Meunier et al., 2003; Hiroi et al., 2004). In contrast to sugars, no significant difference was found in the preference index between $D G s \alpha^{\mathrm{R} 19} /+$ and control flies with any bitter substances tested and at any concentrations (Fig. $2 \mathrm{~B}$ ). This finding indicates that the behavioral response to bitter substances was not impaired in the heterozygous $D G s \alpha$-null mutant.

We next examined the dose-response relationship between the amount of intake and the trehalose concentration in $D G s \alpha^{\mathrm{R} 19} /+$. Although the trehalose intake in the mutant increased with the trehalose concentration, the amount of intake at $10,20,40$, and $80 \mathrm{~mm}$ trehalose in $D G s \alpha^{\mathrm{R} 19} /+$ flies was significantly lower than that at the corresponding concentration in control flies (Fig. $2 C$, open columns, $p<0.05$, asterisks). We also

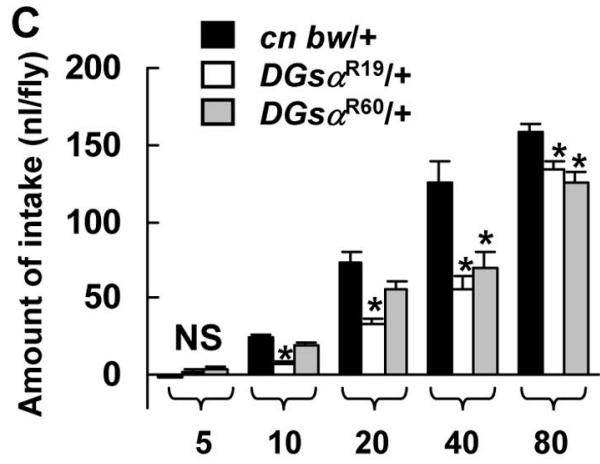

Trehalose concentration $(\mathrm{mM})$

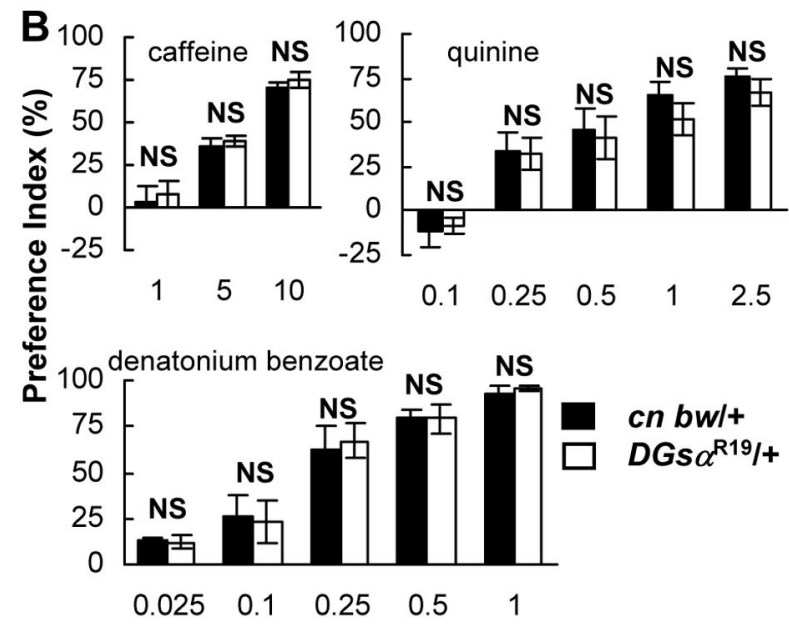

Figure 2. Taste sensitivities to various stimulants in heterozygous $D G s \alpha$-null mutants. Each column represents the mean \pm SEM of 10 experiments. $A$, The amount of intake of $20 \mathrm{~mm}$ trehalose, $5 \mathrm{~mm}$ sucrose, $20 \mathrm{~mm}$ fructose, and $20 \mathrm{~mm}$ glucose in $\mathrm{cm} b w /+$ significantly lower than that in $c n b w /+$ flies (asterisks, $p<0.05$ ). $\boldsymbol{B}$, The preference indexes for the control solution over bitter (filled columns) and $D G s \alpha^{\mathrm{R} 19} /+$ (open columns). The control solution used for quinine and denatofound in the preference indexes between $D G S \alpha^{\mathrm{R} 19} /+$ and $c n b w /+$ flies at any concentrations ( $\left.p>0.05\right)$. NS, Not significant. C, The amount of intake at 10,20,40, and $80 \mathrm{~mm}$ trehalose in $D G s \alpha^{\mathrm{R} 19} /+$ flies (open columns) and the amount of intake at 40 and $80 \mathrm{~mm}$ trehalose in $D G s \alpha^{\mathrm{R} 60} /+$ flies (shaded columns) was significantly lower than in c $b w /+$ flies (filled columns). Asterisks indicate a statistical difference between the control and the heterozygous mutant $(p<0.05)$. $D$, The amount of intake at 20 and $40 \mathrm{~mm}$ trehalose in Gs27; DGs $\alpha^{\mathrm{R} 19} /+$ flies (shaded columns) was not different from in cn bw/ + flies (filled columns, $p>0.05$ ), but significantly higher than that in $D G s \alpha^{\mathrm{R} 19} /+$ flies (open columns, asterisks, $p<0.05$ ).

measured the amount of intake in another heterozygous $D G s \alpha$ null mutant, $D G s \alpha^{\mathrm{R} 60} /+$ (Wolfgang et al., 2001), and found that those at 40 and $80 \mathrm{~mm}$ trehalose in $D G s \alpha^{\mathrm{R} 60} /+$ flies were significantly lower than those at the corresponding concentrations in control flies (Fig. $2 C$, shaded columns, $p<0.05$, asterisks). The depressed trehalose intake in $D G s \alpha^{\mathrm{R} 19} /+$ flies was rescued by the $G s 27$ construct that contains the entire DGs $\alpha$ gene (Wolfgang et al., 2001) (Fig. 2D, shaded columns). These results indicate that the behavioral responses to trehalose in heterozygous DGs $\alpha$-null mutants were impaired.

\section{Transgene DGs $\alpha$ expressed in Gr5a-GRNs rescues impairment of trehalose intake}

To determine whether the depressed trehalose intake in heterozygous $D G s \alpha$-null mutants is attributable to a defect of trehalose response in the Gr5a-GRNs, we expressed the wild-type DGs $\alpha$ 


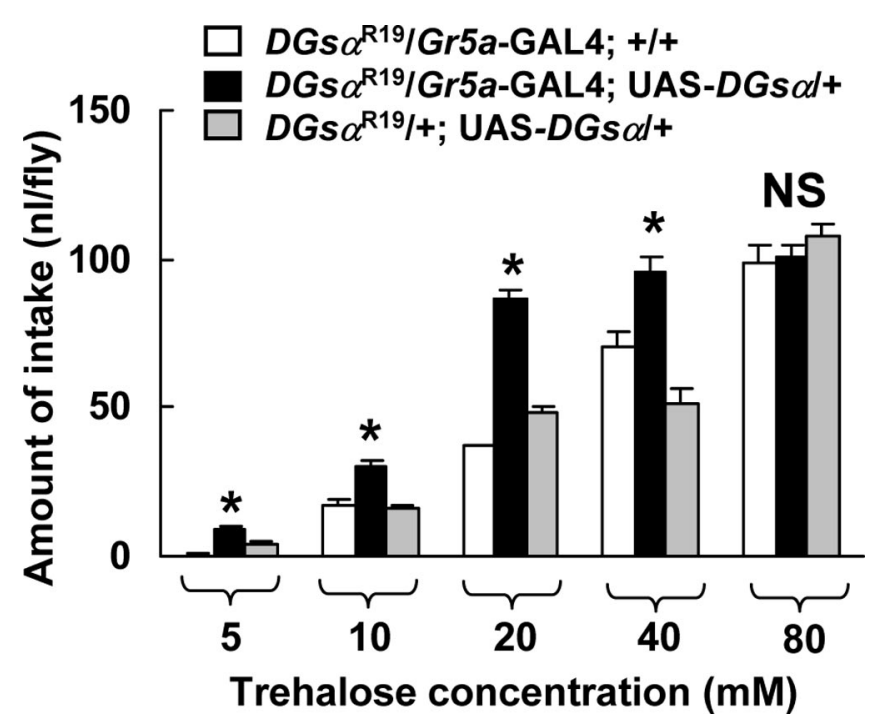

Figure 3. The dose-response relation between the amount of intake and the trehalose concentration in DGs $\alpha^{\mathrm{R} 19} /+$ carrying both Gr5a-GAL4 and UAS-DGs $\alpha$, and $D G s \alpha^{\mathrm{R} 19} /+$ carrying either Gr5a-GAL4 or UAS-DGs $\alpha$ alone. Each column represents the mean \pm SEM of 10 experiments. A significant difference was found between $D G s \alpha{ }^{\mathrm{R} 19} /+$ flies carrying both $G r 5 a$ GAL4 and UAS-DGs $\alpha$ (filled columns) and DGs $\alpha^{\mathrm{R} 19} /+$ flies carrying either Gr5a-GAL4 (open columns) or UAS-DGs $\alpha$ (shaded columns) alone at 5, 10, 20, and $40 \mathrm{~mm}$ (asterisks, $p<0.05$ ). NS, Not significant.

gene exclusively in those neurons in $D G s \alpha^{\mathrm{R} 19} /+$ flies using the GAL4/UAS system. The amount of trehalose intake in $D G s \alpha^{\mathrm{R} 19} /+$ flies carrying both Gr5a-GAL4 and UAS-DGs $\alpha$ was significantly higher than that in $D G s \alpha^{\mathrm{R} 19} /+$ flies carrying either Gr5a-GAL4 or UAS-DGs $\alpha$ alone at 5, 10, 20, and $40 \mathrm{~mm}$ trehalose (Fig. 3, $p<0.05$ ), indicating that depressed trehalose-intake in $D G s \alpha^{\mathrm{R} 19} /+$ flies is rescued by expression of wild-type $D G s \alpha$ exclusively in the Gr5a-GRNs.

$D G s \alpha$ is functionally involved in trehalose-induced electrical responses in sugar-sensitive GRNs

To further confirm the involvement of $D G s \alpha$ in the trehalose response in Gr5a-GRNs, we studied their electrical responses to trehalose in heterozygous $D G s \alpha$-null mutants. It is known that sugar, water, low concentrations of salt and bitter/high concentrations of salt stimuli induce the responses in corresponding four types of GRNs in an L-type chemosensillum (Fujishiro et al., 1984; Hiroi et al., 2004). When an L-type chemosensillum was stimulated with water, a single train of monophasic spikes was observed (Fig. 4A2, expanded trace on the left. In Fig. 4A1, monophasic spikes are marked with asterisks). In contrast, when the chemosensillum was stimulated with $20 \mathrm{~mm}$ trehalose, two kinds of spikes, monophasic and biphasic ones, were observed (Fig. 4A2, an expanded trace on the right is biphasic. In Fig. 4A1, biphasic spikes are marked with dots). The biphasic spikes are most likely to be generated in sugar-sensitive GRNs, whereas monophasic spikes originate from water-sensitive GRNs, because only monophasic spikes were observed when the chemosensillum was stimulated with water and the frequency of monophasic spikes were slightly decreased ( 15.0 spikes/200 ms at $0 \mathrm{~mm}$ treha-

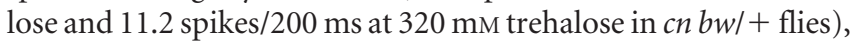
whereas that of biphasic spikes increased with the trehalose concentration (Fig. 4A1). When the chemosensillum in Gr5a-null mutant flies [Gr5a $a^{\Delta \mathrm{EP} 19}$ (Ueno et al., 2001)] was stimulated with trehalose, the number of biphasic spikes was very low (data not shown), in accord with a previous finding that the number of sugar-sensitive GRNs in $G r 5 a^{\Delta \mathrm{EP} 19}$ is extremely low (Dahanukar et al., 2001). Therefore, we considered that the biphasic spikes that we recorded in these experiments were generated in sugarsensitive Gr5a-GRNs.

It is possible that the monophasic spikes are not only generated by water-sensitive GRNs but also by GRNs sensitive to low concentrations of salt because we always had $7.5 \mathrm{~mm} \mathrm{KCl}$ in the recording solution as an electrical conductor. However, we observed two-types of spikes, monophasic and biphasic spikes, in 20 $\mathrm{mm} \mathrm{NaCl}$ solution. When the $\mathrm{NaCl}$ concentration was increased to $100 \mathrm{~mm}$, the frequency of monophasic spikes decreased slightly, whereas that of biphasic spikes dramatically increased (data not shown). Thus, we believe that the monophasic spikes are generated by water-sensitive GRNs.

All transformants responded to trehalose (Fig. 4A1). However, the response in $D G s \alpha^{\mathrm{R} 19} /+$ flies carrying both Gr5a-GAL4 and UAS-DGs $\alpha$ was significantly stronger than the responses in the other genotypes at 5, 20, and $80 \mathrm{~mm}$ trehalose (Fig. $4 \mathrm{~B}$, asterisks, $p<0.05)$. This result indicates that the electrical responses to trehalose in $G r 5 a-G R N s$ in $D G s \alpha^{\mathrm{R} 19} /+$ flies are rescued by the expression of wild-type $D G s \alpha$ exclusively in Gr5a-GRNs.

The amount of trehalose intake in $D G s \alpha^{\mathrm{R} 19} /+$ flies carrying both Gr5a-GAL4 and UAS-DGs $\alpha$ was lower at $80 \mathrm{~mm}$ trehalose and higher at $5 \mathrm{~mm}$ trehalose than that in $c n b w /+$ flies (Fig. $5 A$ ). In contrast, the electrical response in $D G s \alpha^{\mathrm{R} 19} /+$ flies carrying both $G r 5 a$-GAL4 and UAS-DGs $\alpha$ was not significantly different from the responses in $c n b w /+$ flies at 5, 20, 80, and $320 \mathrm{~mm}$ trehalose (Fig. 5B). To account for this discrepancy, we suggest that DGs $\alpha$ in non-Gr5a cells, for example, CNS neurons, is also involved in the behavioral response.

Neuronal electrical responses to water were not different between $D G s \alpha^{\mathrm{R} 19} /+$ flies carrying both Gr5a-GAL4 and UAS$D G s \alpha$ and $c n b w /+$ flies (Fig. $5 B$ ). This result indicates that the response to water in water-sensitive GRNs is not impaired in $D G s \alpha^{\mathrm{R} 19} /+$ flies.

Trehalose response in double mutants of $D G s \alpha^{\mathrm{R} 19} /+$ and $G r 5 a$-null was not rescued by exogenous wild-type $D G s \alpha$ The depressed electrophysiological trehalose response in $D G s \alpha^{\mathrm{R} 19} /+$ flies was rescued when exogenous $D G s \alpha$ was expressed in Gr5a-GRNs (Figs. 4B, 5B). However, it is still possible that expressing exogenous $D G s \alpha$ nonspecifically enhanced the activity of sugar-sensitive Gr5a-GRNs. Furthermore, it is also possible that the increase of biphasic spike frequency in $D G s \alpha^{\mathrm{R} 19} /+$ flies carrying both Gr5a-GAL4 and UAS-DGs $\alpha$, compared with $D G s \alpha^{\mathrm{R} 19} /+$ flies, is attributable to recruitment of non-sugar-sensitive Gr5a-GRNs, because it is known that L-type chemosensilla contain multiple Gr5a-GRNs (Thorne et al., 2004), and another report suggests that some of Gr5a-GRNs serve as salt-sensitive GRNs (Wang et al., 2004). To rule out these possibilities, we generated double mutants of $D G s \alpha^{\mathrm{R} 19}$ and $G r 5 a^{\Delta \mathrm{EP} 19}$. Gr5a $a^{\Delta \mathrm{EP} 19}$ is a Gr5a-null allele and has an extremely low sensitivity to trehalose, as mentioned above (Dahanukar et al., 2001; Ueno et al., 2001). If exogenous $D G s \alpha$ nonspecifically enhanced the activity of sugar-sensitive Gr5a-GRNs or enhanced the activity of salt-sensitive Gr5a-GRNs, exogenous DGs $\alpha$ should also have increased the number of biphasic spikes even in the $G r 5 a$-null allele.

The behavioral responses to trehalose in three strains of double mutants were very low and not significantly different among them $(p>0.05)$ (Fig. 6A), nor were electrical responses robust. The response to $20 \mathrm{~mm}$ trehalose was not detected in sugarsensitive GRNs, and the response to $320 \mathrm{~mm}$ trehalose was at a low level in all strains of flies carrying $G r 5 a^{\Delta \mathrm{EP} 19}$ (Fig. $6 B$ ). No 
significant differences were detected at any concentrations $(5 \sim 320 \mathrm{~mm})$ of trehalose among three transformants $(p>0.05)$ (Fig. 6B). These results indicate that the expression of exogenous wild-type $D G s \alpha$ enhances neither the activity of sugar-sensitive Gr5a-GRNs nonspecifically nor the activity of saltsensitive Gr5a-GRNs and suggest that $\mathrm{DGs} \alpha$ involved in the downstream transduction pathway of Gr5a in sugar-sensitive-GRNs.

RNAi for DGs $\alpha$ depresses the expression of DGs $\alpha$ in $G r 5 a-G R N s$ and both behavioral and

electrophysiological trehalose responses To confirm that $D G s \alpha$ is involved in the trehalose response in sugar-sensitive Gr5a-GRNs, we used the RNAi technique. With the Western blotting analysis, we confirmed that the expression level of $D G s \alpha$ is depressed in the brain of larvae by the UAS-DGs $\alpha$ RNAi construct (Fig. 7A). Next, we immunohistochemically examined the effect of expressing the DGs $\alpha$ RNAi construct in GRNs. In flies in which GFP was expressed in Gr5a-GRNs (Gr5a-GAL4/UAS-mCD8::GFP), Gr5aGRNs were stained as yellow by simultaneous staining with anti-GFP antibody (green) and anti-Gs $\alpha$ peptide antiserum (red) (Fig. 7B, top panel). In contrast, in flies in which $D G s \alpha$ RNAi was coexpressed (Gr5a-GAL4/UAS-mCD8::GFP/UAS-DGs $\alpha$ RNAi), the majority of Gr5a-GRNs showed green fluorescence and weak yellow fluorescence (Fig. $7 B$, bottom panel). In either type of flies, non-Gr5a GRNs showed red immunofluorescence (Fig. $7 B$, top and bottom panels). Thus, we confirmed that introducing the $D G s \alpha$ RNAi construct suppressed $D G s \alpha$ expression in Gr5a-GRNs.

The behavioral responses to trehalose in two strains of transgenic flies carrying both Gr5a-GAL4 and UAS-DGs $\alpha$ RNAi constructs (\#32-1 and \#20-1) were significantly lower than in flies carrying either the Gr5a-GAL4 or the UAS-DGs $\alpha$ RNAi construct $[p<0.05$ (Fig. 7C) in Gr5aGAL4/UAS-DGs $\alpha$ RNAi (\#32-1) at 10, 20, 40 , and $80 \mathrm{~mm}$ and in Gr5a-GAL4/UAS-

$D G s \alpha$ RNAi (\#20-1) at 20 and $40 \mathrm{~mm}$ ].

Gr66a is expressed in bitter-sensitive GRNs and is not coexpressed with $G r 5 a$, although natural ligands for Gr66a are not known (Thorne et al., 2004; Wang et al., 2004). The trehalose responses in transgenic flies carrying Gr66a-GAL4 and UAS-DGs $\alpha$ RNAi were not impaired (Fig. 7C). Hence, we conclude that the reduction of trehalose responses in two strains of transgenic flies described above is the result of the inhibitory effects of $D G s \alpha$ RNAi specifically in Gr5a-GRNs.

The electrophysiological responses in sugar-sensitive Gr5a-

A1

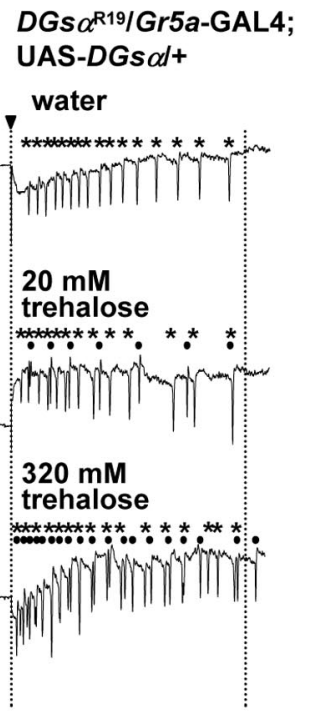

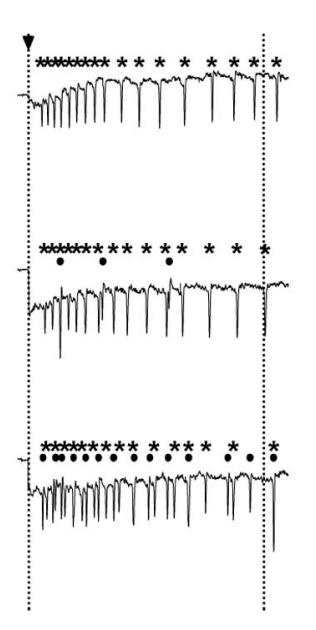

DGs $\alpha^{R 19 / G r 5 a-G A L 4 ; ~+/+~}$
$D G s \alpha^{R 19 /+; ~ U A S-D G s ~} \alpha l+$

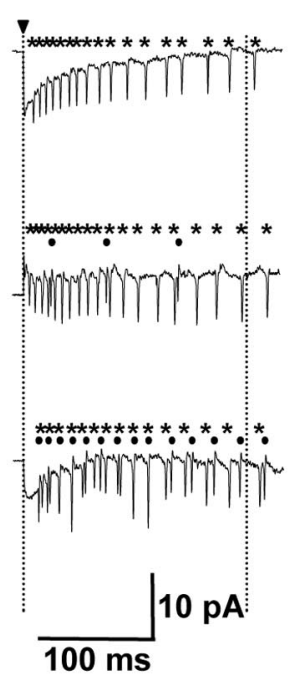

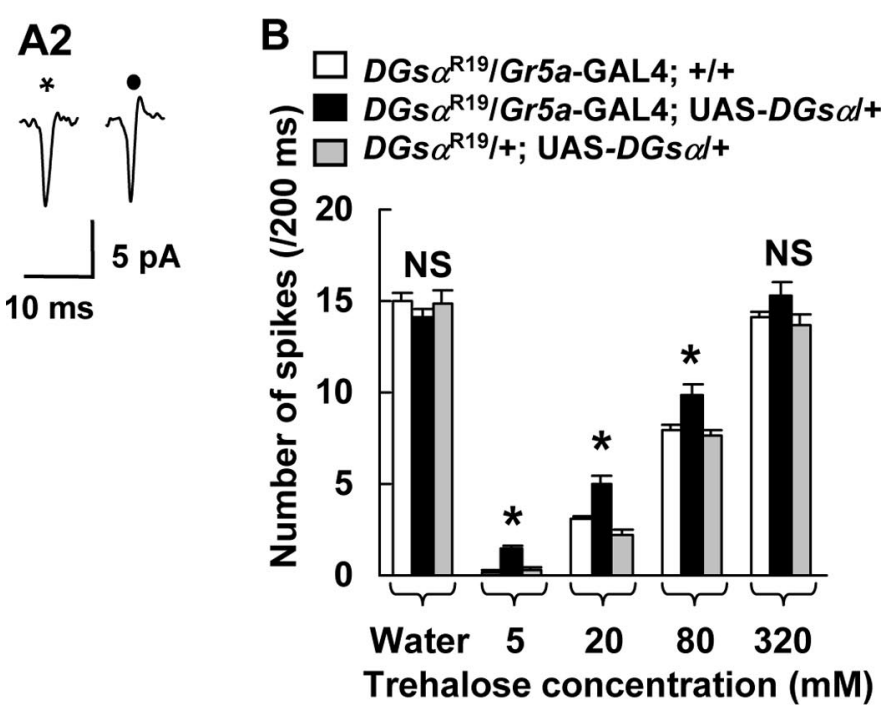

Figure 4. Electrical responses in GRNs to trehalose in heterozygous $D G s \alpha$-null mutants. A1, Typical responses in L-type chemosensilla to water ( $7.5 \mathrm{~mm} \mathrm{KCI}), 20$ and $320 \mathrm{~mm}$ trehalose solutions in $D G 5 \alpha^{\mathrm{R} 19} /+$ flies carrying both Gr5a-GAL4 and UAS-DGS $\alpha$ (left three traces) and DGs $\alpha^{\mathrm{R} 19} /+$ carrying either Gr5a-GAL4 (middle three traces) or UAS-DGs $\alpha$ (right three traces) alone. Spikes marked with asterisks and dots were counted separately as a measure of magnitude of the response in a water-sensitive GRN (asterisks) and in a sugar-sensitive GRN (dots) during a $200 \mathrm{~ms}$ period starting from the onset of stimulus (period between two vertical dotted lines). Arrowheads indicate the onset of stimulation. A2, Two expanded traces represent typical spikes from a water-sensitive GRN (asterisk) and a sugar-sensitive GRN : $\boldsymbol{B}$, Each column represents the number of spikes in a sugar-sensitive GRN during a $200 \mathrm{~ms}$ period starting from the onset of stimulus. A significant difference was found between $D G s \alpha^{\mathrm{R} 19} /+$ flies carrying both Gr5a-GAL4 and UAS-DGs $\alpha$ (filled columns) and DGs $\alpha^{\mathrm{R} 19} /+$ flies carrying either Gr5a-GAL4 (open columns) or UAS-DGs $\alpha$ (shaded columns) alone at 5, 20, and $80 \mathrm{~mm}$ trehalose (asterisks, $p<0.05$ ). Columns marked "Water" represent the response to water of a water-sensitive GRN. Each column represents the mean \pm SEM of 15 samples. NS, Not significant.
GRNs at 5, 20, and $80 \mathrm{~mm}$ trehalose in transgenic flies carrying both Gr5a-GAL4 and UAS-DGs $\alpha$ RNAi constructs were significantly lower than those in transgenic flies carrying either Gr5aGAL4 or UAS-DGs $\alpha$ RNAi alone (Fig. 7D, $p<0.05$ ). These findings indicate that the reduced expression level of $D G s \alpha$ in the transformant carrying both Gr5a-GAL4 and UAS-DGs $\alpha$ RNAi constructs depressed trehalose responses in sugar-sensitive Gr5a-GRNs. 


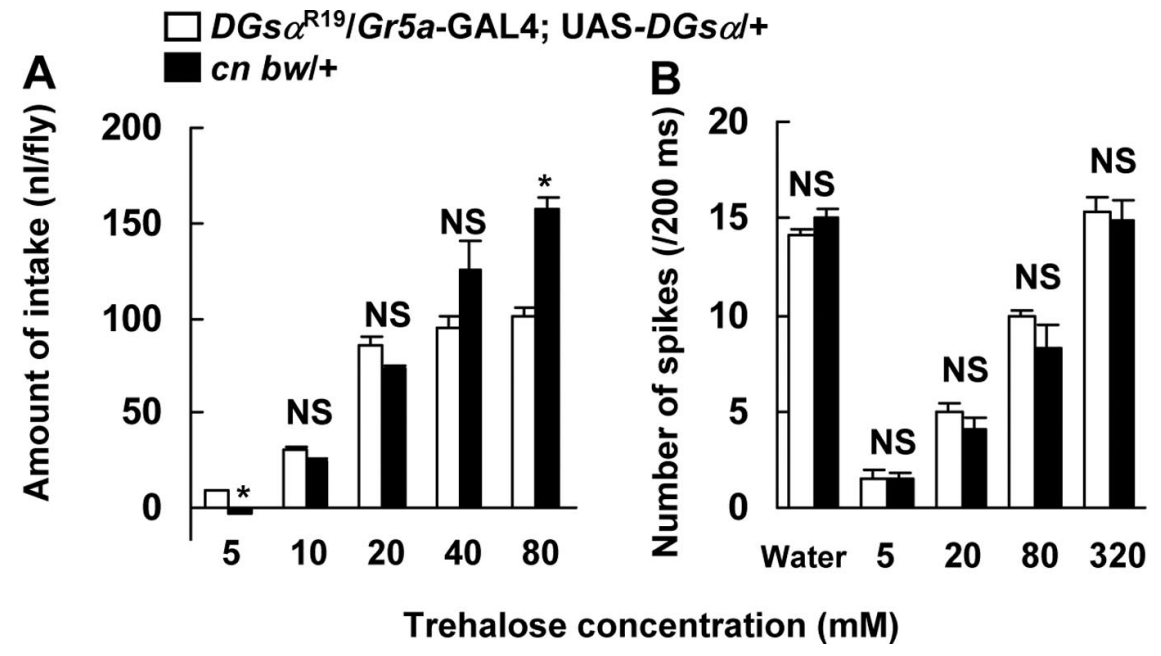

Figure 5. The dose-response relations between behavioral responses and trehalose concentration $(\boldsymbol{A})$, and electrical responses and trehalose concentration (B) in $D G s \alpha^{\mathrm{R} 19} / G r 5 a-G A L 4 ;$ UAS-DGs $\alpha /+$ and $c n b w /+$ flies. $\boldsymbol{A}$, The data in Figure 3 are replotted for DGs $\alpha^{\mathrm{R} 19} / \mathrm{Gr} 5 a$-GAL4; UAS-DGs $\alpha /+$ (open columns) and the data in Figure 2C are replotted for $\mathrm{cn}$ bw/ + flies (filled columns). A significant difference was found between $D G s \alpha^{\mathrm{R} 19} / G r 5 a-G A L 4 ;$ UAS-DGs $\alpha /+$ and $c n$ bw/ + flies at 5 and $80 \mathrm{~mm}$ trehalose (asterisks, $p<0.05$ ). Each column represents mean \pm SEM of 10 samples. $\boldsymbol{B}$, The data in Figure $4 B$ are replotted for $D G s \alpha^{\mathrm{R} 19} /$ Gr5a-GAL4; UAS-DGs $\alpha /+$ flies (open columns). The filled columns represent the electrical responses to trehalose in $c n$ $b w /+$ flies. Columns marked "Water" represent the response to water in a water-sensitive GRN. Each column represents mean \pm SEM of 15 samples. No significant difference was found between the two mutant flies ( $p>0.05)$. NS, Not significant.

A
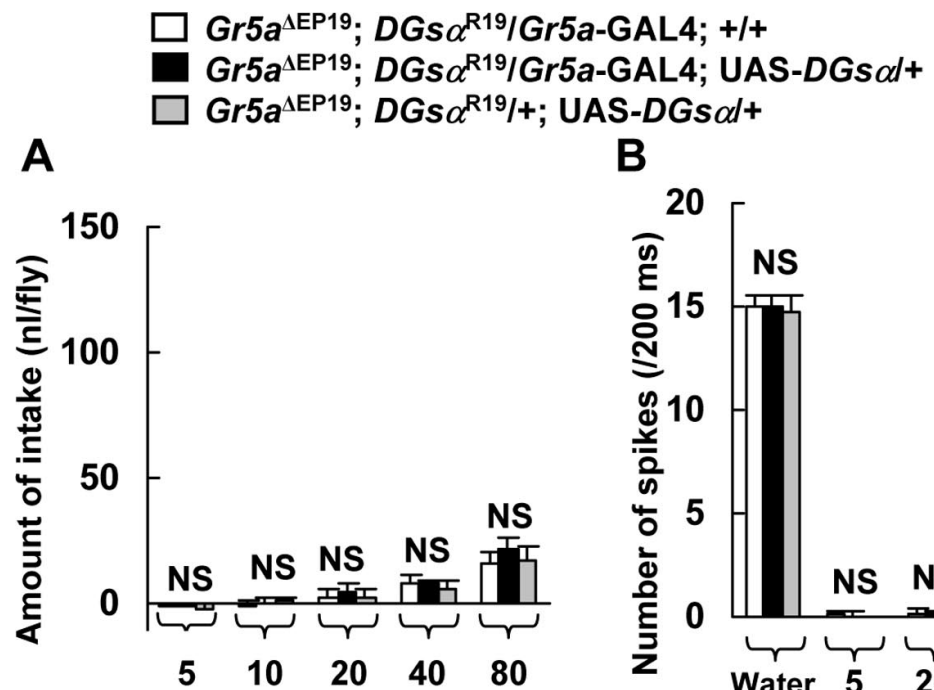

$B$

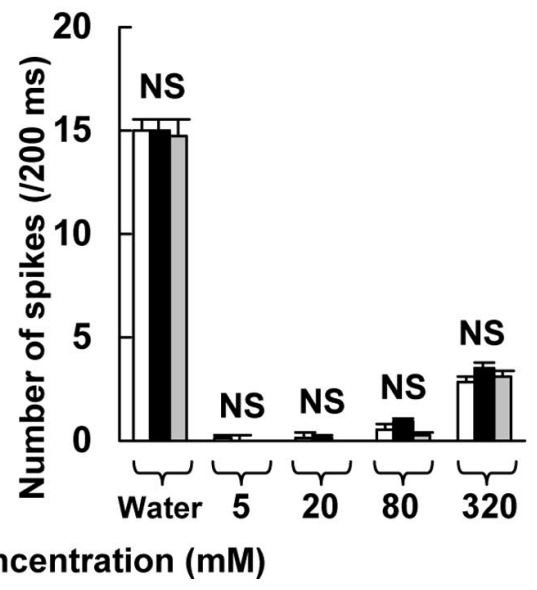

Figure 6. The dose-response relations between behavioral responses and trehalose concentration $(\boldsymbol{A})$ and electrical responses and trehalose concentration $(\boldsymbol{B})$ in a $D G s \alpha^{\text {R19 }}$ and Gr5a $a^{\Delta \text { EP19 }}$ double mutant. Wild-type DGs $\alpha$ was expressed in Gr5a-GRNs in $D G s \alpha^{\mathrm{R} 19} /+$ in the Gr5a-null background using the GAL4/UAS system (Gr5a $a^{\Delta \text { EP19; }}$ DGs $\alpha^{\mathrm{R} 19} /$ Gr5a-GAL4; UAS-DGs $\left.\alpha /+\right)$.

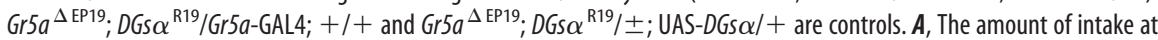
$5,10,20,40$, and $80 \mathrm{~mm}$ trehalose in Gr5a ${ }^{\Delta \text { EP19. }}$ DGs $\alpha^{\text {R19 }} /$ Gr5a-GAL4; + / (open columns), Gr5a $a^{\Delta \text { EP19 }}$; DGs $\alpha^{\text {R19 } / G r 5 a-G A L 4 ; ~}$ UAS-DGs $\alpha /+$ (filled columns), and Gr5a ${ }^{\Delta \mathrm{EP} 19} ; D G s \alpha^{\mathrm{R} 19} / \pm ;$ UAS-DGs $\alpha /+$ (shaded columns). Each column represents the mean \pm SEM of 10 experiments. No significant difference was found among three double mutants at any concentrations $(p>$ 0.05). $\boldsymbol{B}$, The number of spikes in 5, 20,80, and $320 \mathrm{~mm}$ trehalose solutions from sugar-sensitive GRNs during a $200 \mathrm{~ms}$ period starting from the onset of stimulus in Gr5a $a^{\Delta \text { EP19. }} ; D G s \alpha^{\text {R19 }} /$ Gr5a-GAL4; + + (open columns), Gr5a $a^{\Delta \text { EP19. }}$ DGs $\alpha^{\text {R19 } / G r 5 a-G A L 4 ; ~}$ UAS-DGs $\alpha /+$ (filled columns), and Gr5a ${ }^{\Delta \text { EP19 }} ; D G s \alpha^{\text {R19 }} / \pm ;$ UAS-DGs $\alpha /+$ (shaded columns). No significant difference was found among three double mutants at any concentrations $(p>0.05)$. Columns marked "Water" represent the response to water of a water-sensitive GRN. Each column represents the mean \pm SEM of 15 samples. NS, Not significant.

Trehalose response in a norpA-null mutant In addition to the involvement of $D G s \alpha$ in the $G r 5 a$-mediated trehalose-signaling pathway, phospholipase C (PLC) might also play a role in this pathway (Koganezawa and Shimada, 2002;
Chyb et al., 2003). To test this possibility, we used norpA, which encodes PLC, and measured the amount of trehalose intake in a norpA-null allele, norp $A^{\mathrm{P} 24}$ (Pearn et al., 1996). The amount of trehalose intake was not different between norp $A^{\mathrm{P} 24}$ and control flies at any concentrations, except at $0.8 \mathrm{~mm}$, at which level trehalose intake was significantly higher in norp $A^{\mathrm{P} 24}$ than in the control (Fig. $8 A$, asterisk).

The electrophysiological responses in sugar-sensitive Gr5a-GRNs in norp $A^{\mathrm{P} 24}$ and control flies were not significantly different at any concentrations of trehalose $(0.8,4,20,100$, and $400 \mathrm{~mm}$ ) (Fig. $8 B, p>$ $0.05)$. These findings indicate that the behavioral and electrophysiological responses to trehalose in norp $A^{\mathrm{P} 24}$ flies were not impaired, although the expression of PLC encoded by norpA is eliminated in norp $A^{\mathrm{P} 24}$ flies (Pearn et al., 1996). Thus, we conclude that norpA does not mediate trehalose-taste signaling.

\section{Discussion}

In this study, we conclude that a specific $\mathrm{G} \alpha$, Gs $\alpha$, which is encoded by $D G s \alpha$, is involved in the sugar response in Gr5aGRNs, and we suggest that the DGs $\alpha$ mediating transduction pathway is coupled to Gr5a and processes sugar signaling in Drosophila.

After identifying Drosophila gustatory receptors as GPCRs, Clyne et al. (2000) postulated that the transduction pathway of taste in Drosophila is mediated by G-proteins. Recently, a $\gamma$ subunit of the G-protein encoded by $G \gamma 1$ was reported to be required for sugar perception (Ishimoto et al., 2005). Although this finding confirms the hypothesis that the sugargustatory receptors, including Gr5a, are coupled to G-proteins, it does not specify the downstream pathway for sugar-taste signaling in Drosophila. Our finding that Gs $\alpha$ is involved in sugar perception in Drosophila strongly suggests that the cAMP transduction pathway is involved in sugar-taste signaling. Although in vertebrates it has not been established which $\mathrm{G} \alpha$ mediates sugar-taste signaling, this is the first demonstration in Drosophila that $\mathrm{Gs} \alpha$ is involved in sugar-taste signaling in vivo.

The responses to sugars, i.e., trehalose, sucrose, fructose, and glucose, were impaired in the heterozygous $D G s \alpha$-null mutants (Fig. 2A), although it is known that $\mathrm{Gr} 5 \mathrm{a}$ is narrowly tuned to trehalose (Chyb et al., 2003). Hence, it is possible that the other sugar receptors, yet to be identified, are also coupled to $D G s \alpha$ and have their own transduction pathway.

We found that the behavioral responses to trehalose in het- 
erozygotes of two $D G s \alpha$-null alleles $\left[D G s \alpha^{\mathrm{R} 19}\right.$ and $D G s \alpha^{\mathrm{R} 60}$ (Wolfgang et al., 2001)] were lower than in the control (Fig. $2 C)$. The trehalose intake in $D G s \alpha^{\mathrm{R} 19} /+$ was more severely depressed than in $D G s \alpha^{\mathrm{R} 60} /+$. This difference could arise from an additional mutation in $D G s \alpha^{\mathrm{R} 19}$ (Wolfgang et al., 2001). However, the depression of trehalose intake in $D G s \alpha^{\mathrm{R} 19} /+$ was completely rescued by expressing exogenous $D G s \alpha$ in $G s 27$ (Fig. 2D) or in Gr5a-GAL4/UAS-DGs $\alpha$ (Fig. 3). These results indicate that the depression of trehalose intake in $D G s \alpha^{\mathrm{R} 19} /+$ flies is attributable to the $D G s \alpha^{\mathrm{R} 19}$ mutation. It is unclear at this moment what is causing the difference between $D G s \alpha^{\mathrm{R} 19} /+$ and $D G s \alpha^{\mathrm{R} 60} /+$.

It is possible that $D G s \alpha$ is not required for trehalose-taste signaling but is necessary for development of sugar-sensitive GRNs, and the low trehalose responses in transgenic flies expressing DGs $\alpha$ RNAi (Gr5a-GAL4/UAS-DGs $\alpha$ RNAi) were caused by developmental defects of the neurons. For example, $D G s \alpha$ might be required for expression of $G r 5 a$ or ion channels. However, the response induced by $320 \mathrm{~mm}$ trehalose in those flies was not different from that in the control (Fig. $7 D)$. This result indicates that the sugarsensitive GRNs in the transgenic flies are fully equipped with the molecular machinery for the maximal trehalose response. Thus, we suggest that the developmental effect of $D G s \alpha$ on sugar-sensitive GRNs is not a major contributor to the observed RNAi effect.

In the RNAi analysis, the responses to trehalose in the transgenic DGs $\alpha$ RNAiexpressing flies were reduced but not eliminated (Fig. 7C,D). The residual responses in the transgenic flies might be attributable to the residual DGs $\alpha$ protein, because the $D G s \alpha$ expression was not completely eliminated in the transgenic flies (Fig. $7 A, B$ ). However, it is possible that a non-DGs $\alpha$ transduction pathway is also involved in sugar perception. A previous report suggested that the Gq/PLCmediated pathway is involved in the Gr5ainitiated signaling pathway in the S2 cell line (Chyb et al., 2003), and another report showed that $d G q$, a homolog of mammalian Gq, and norp $A$ are expressed in the labelum (Talluri et al., 1995; Koganezawa and Shimada, 2002). It is, therefore, possible that the PLC system is synergistically contributing to sugar-taste perception together with the Gr5a/DGs $\alpha$ signaling pathway. Using a norpA-null allele, norp $A^{\mathrm{P} 24}$, we tested this possibility. We found that the amount of trehalose intake was not significantly different between norp $A^{\mathrm{P} 24}$ and control flies at most concentrations tested (Fig. $8 A$ ). Furthermore, the electrophysiological response to tre-

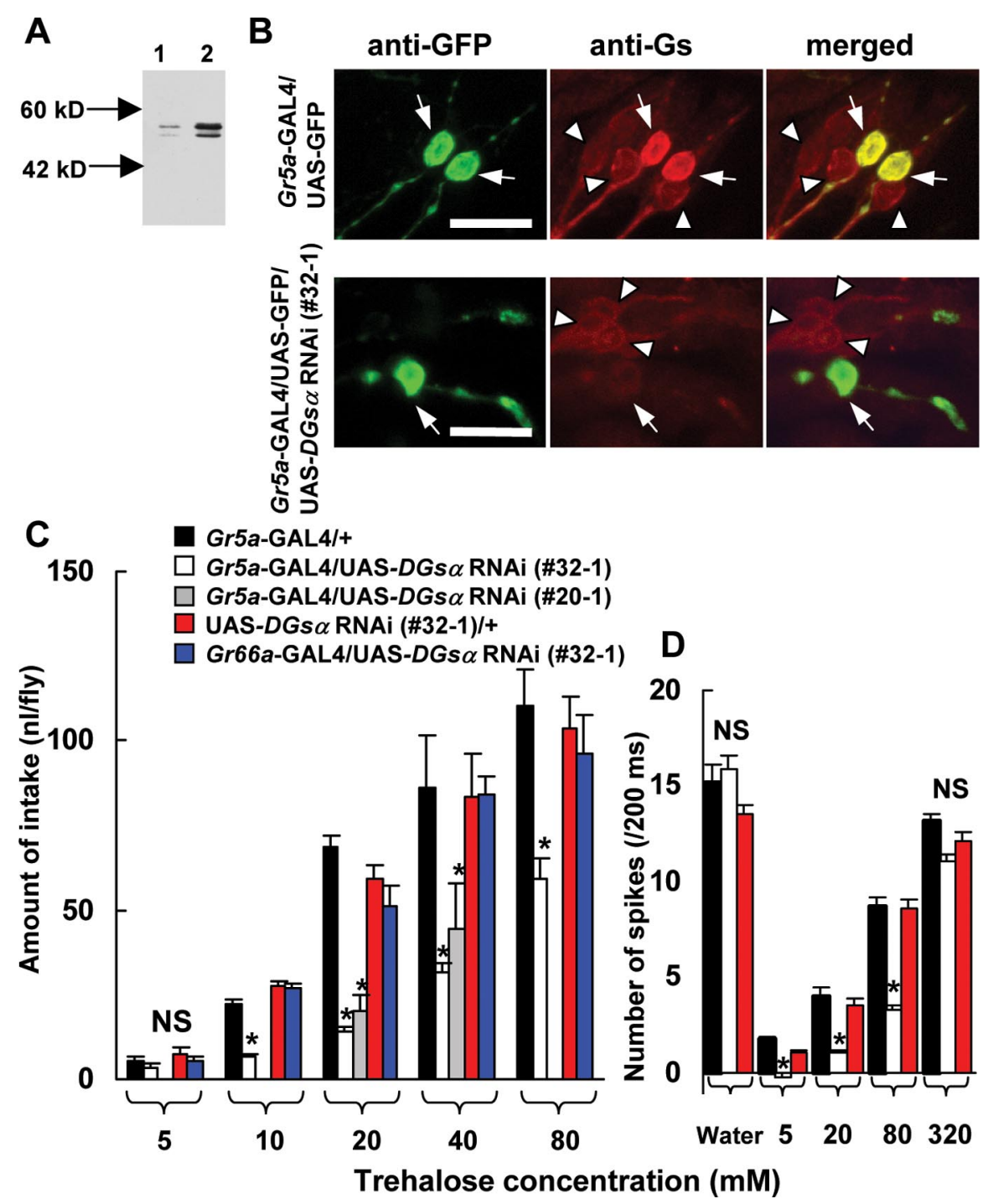

Figure 7. The effects of $D G S \alpha$ RNAi on immunoreactivities in larval CNS and GRNs $(A, B)$ and on behavioral ( $C$ and electrophysiological responses (D) to trehalose. $\boldsymbol{A}$, Immunoblot of the DGs $\alpha$ protein obtained from larval CNS. Lane 1, DGs $\alpha$ RNAi expressed in larval CNS (1407-GAL4/UAS-DGs $\alpha$ RNAi). Lane 2, Control (+/UAS-DGs $\alpha$ RNAi). The antiserum recognized short and long forms of DGs $\alpha$ (Quan and Forte, 1990). $\boldsymbol{B}$, Effects of $D G s \alpha$ RNAi expressed in Gr5a-GRNs. Control images stained with anti-GFP antibody (top left panel) and with anti-Gs $\alpha$ peptide antiserum (top middle panel), and merged (top right panel) in a transgenic fly expressing GFP in Gr5a-GRNs. The bottom panels are corresponding images in a transgenic fly expressing GFP and DGs $\alpha$ RNAi in Gr5a-GRNs. Arrows indicate Gr5a-GRNs. In the top panels, two Gr5a-GRNs (arrows) show immunofluorescence against GFP (left panel) as well as against Gs $\alpha$ (middle panel) resulting in yellow (right panel). In the bottom panels, in which DGs $\alpha$ RNAi was expressed, anti-Gs $\alpha$ fluorescence was very dim in a Gr5a-GRN (arrow in middle panel), resulting in green in a merged image (arrow in right panel). Immunoreactivities against Gs $\alpha$ in non-Gr5a GRNs (arrowheads) of both transgenic flies are visible. Scale bars, $10 \mu \mathrm{m}$. C, The amount of trehalose intake in DGs $\alpha$ RNAi-expressing flies [Gr5a-GAL4/ UAS-DGs $\alpha$ RNAi (\#32-1), open columns] was lower than in controls (black and red columns) at 10, 20, 40, and 80 mm trehalose (asterisks, $p<0.05$ ). The amount of trehalose intake in another DGs $\alpha$ RNAi-expressing strain [Gr5a-GAL4/UAS-DGs $\alpha$ RNAi ( $\# 20-1)$, gray columns] was also lower than in control at 20 and $40 \mathrm{~mm}$ (asterisks, $p<0.05$ ). Each column represents the mean $\pm S E M$ of 10 experiments. № significant difference was found among flies expressing DGs $\alpha$ RNAi in bitter-sensitive GRNs (Gr66a-GAL4/UAS-DGs $\alpha$ RNAi, blue columns) and controls (black and red columns). D, Electrical responses to trehalose in flies expressing DGs $\alpha$ RNAi (Gr5a-GAL4/UAS-DGs $\alpha$ RNAi). $x$ and $y$-axes are the same as Figure 4B. A significant difference was found among flies expressing DGS $\alpha$ RNAi [Gr5a-GAL4/UAS-DGs $\alpha$ RNAi (\#32-1), open columns] and controls (black and red columns) at 5, 20, and 80 mm trehalose (asterisks, $p<0.05$ ). Each column represents the mean \pm SEM of 15 samples.

halose was not significantly different at any concentrations between norp $A^{\mathrm{P} 24}$ and control flies (Fig. 8 B). It is unlikely that PLC encoded by genes other than norp $A$ is involved in this pathway, because PLC activities are completely eliminated in adult heads of norp $A^{\mathrm{P} 24}$ flies (Zhu et al., 1993). Therefore, we suggest that the PLC transduction pathway is not significantly contributing to the sugar response in Drosophila. 


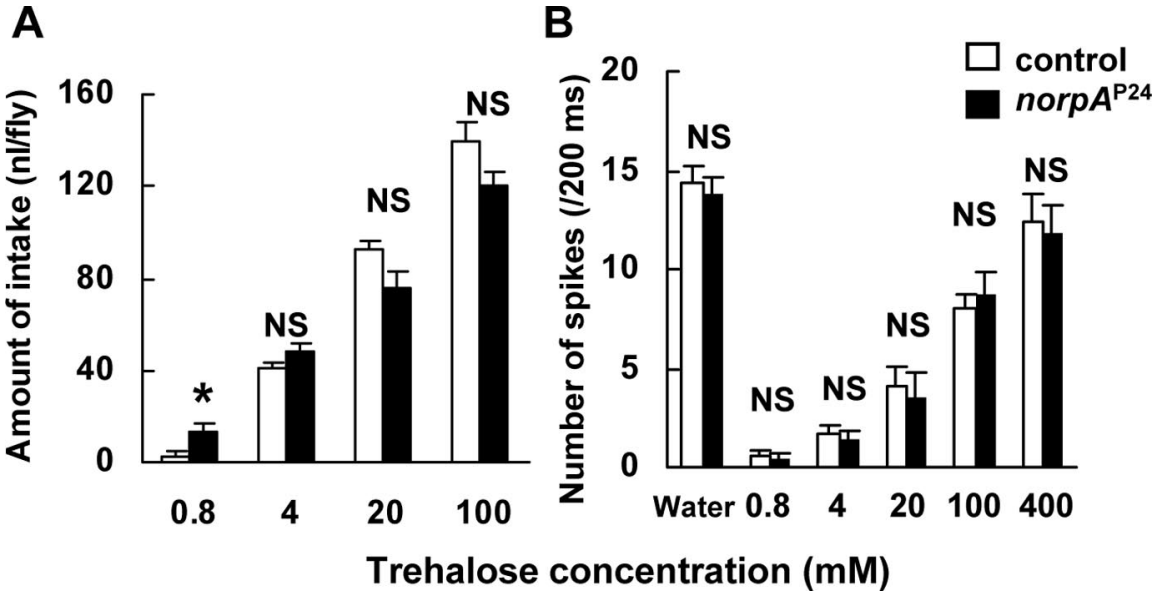

Figure 8. The dose-response relations between behavioral responses and trehalose concentration $(\boldsymbol{A})$, and electrical responses and trehalose concentration $(\boldsymbol{B})$ in norpA $A^{\mathrm{P} 24}$ and control flies. $\boldsymbol{A}$, Each column represents mean \pm SEM of eight samples. A significant difference between norp $A^{\mathrm{P} 24}$ and control flies was found only at $0.8 \mathrm{~mm}$ trehalose (asterisk, $p<0.05$ ). $\boldsymbol{B}$, The number of spikes to $0.8,4,20,100$, and $400 \mathrm{~mm}$ trehalose solutions from sugar-sensitive GRNs during a 200-ms period starting from the onset of stimulus in norpA ${ }^{\mathrm{P} 24}$ and control flies. Columns marked "Water" represent the response to water of a water-sensitive GRN. No significant difference was found between norpA ${ }^{\mathrm{P} 24}$ and control flies. Each column represents mean \pm SEM of 15 samples. tory sugar receptors of the mouse supports involvement of the PLC pathway (Zhang et al., 2003). Additional comparative studies are necessary to elucidate the diversity of molecular mechanisms of sugar-taste signaling in various animals.

\section{References}

Amakawa T, Ozaki M, Kawata K (1990) Effects of cyclic GMP on the sugar taste receptor cell of the fly Phormia regina. J Insect Physiol 36:281-286.

Amrein H, Thorne N (2005) Gustatory perception and behavior in Drosophila melanogaster. Curr Biol 15:R673-R684.

Avenet P, Lindemann B (1987) Patch-clamp study of isolated taste receptor cells of the frog. J Membr Biol 97:223-240.

Bloomquist BT, Shortridge RD, Schneuwly S, Perdew M, Montell C, Steller H, Rubin G, Pak WL (1988) Isolation of a putative phospholipase C gene of Drosophila, norpA, and its role in phototransduction. Cell 54:723-733.

Chyb S, Dahanukar A, Wickens A, Carlson JR (2003) Drosophila Gr5a encodes a taste receptor tuned to trehalose. Proc Natl Acad Sci USA

We found that the behavioral response to $0.8 \mathrm{~mm}$ trehalose in norp $A^{\mathrm{P} 24}$ was higher than control flies (Fig. $8 A$ ), although the electrophysiological responses to $0.8 \mathrm{~mm}$ trehalose were not different between norp $A^{\mathrm{P} 24}$ and control flies (Fig. $8 \mathrm{~B}$ ). These results suggest that norp $A$ is involved in the higher processing of gustatory response, for example, in the CNS. It is known that norpA is expressed in the brain (Zhu et al., 1993).

We found that DGs $\alpha$ is localized not only in Gr5a-GRNs but also in non-Gr5a GRNs ( $\sim 40$ GRNs in a labelum) (Fig. $1 D, E)$. In labela, there are at least four types of GRNs sensitive to sugar, low concentrations of salt, bitter-substances/high concentrations of salt, water, and mechanosensory neurons (Falk et al., 1976; Fujishiro et al., 1984; Meunier et al., 2003; Hiroi et al., 2004). Then, two questions arise: (1) which GRN, other than Gr5a-GRNs, contains DGs $\alpha$ ? (2) Is DGs $\alpha$ in unknown GRNs involved in the taste signaling of GRNs? The behavioral responses to bitter solutions were not different between heterozygous $D G s \alpha$-null mutant and control flies (Fig. 2B), and the behavioral and electrophysiological responses to water were not different among all DGs $\alpha$ strains examined in this study. It is known that salt responses in larvae require amiloride-sensitive channels encoded by $p p k 11$ and ppk19 (Liu et al., 2003), and the low and high concentrations of salt responses do not require $G \gamma 1$ in adult flies (Ishimoto et al., 2005). These findings together with our results suggest that $D G s \alpha$ in non-Gr5a GRNs serves for other signaling than taste or that the non-Gr5a GRNs containing DGs $\alpha$ are mechanosensory neurons. However, because we did not examine the bitter and water responses in the homozygous $D G s \alpha^{\mathrm{R} 19}$ mutant, we cannot rigorously exclude the possibility of whether DGs $\alpha$ is involved in bitter and/or water tastes.

We suggest that, in Drosophila, the Gs-mediated cAMP transduction pathway is the main signaling route in sugar-sensitive GRNs. In contrast, the PLC/ $\mathrm{IP}_{3}$ mediating pathway is involved in sugar-taste signaling in the fleshfly (Boettcherisca peregrina) (Koganezawa and Shimada, 2002) and the guanosine-3', 5' -cyclic monophosphate/nitric oxide pathway in the blowfly (Phormia regina) (Amakawa et al., 1990; Murata et al., 2004). The cAMP pathway may be involved in sugar-taste perception in the frog, rat, and pig (Avenet and Lindemann, 1987; Striem et al., 1989; Naim et al., 1991), whereas a recent study on T1R2/T1R3 gusta-
100 [Suppl 2]:14526-14530.

Clyne PJ, Warr CG, Carlson JR (2000) Candidate taste receptors in Drosophila. Science 287:1830-1834.

Connolly JB, Roberts IJ, Armstrong JD, Kaiser K, Forte M, Tully T, O’Kane CJ (1996) Associative learning disrupted by impaired Gs signaling in Drosophila mushroom bodies. Science 274:2104-2107.

Dahanukar A, Foster K, van der Goes van Naters WM, Carlson JR (2001) A $\mathrm{Gr}$ receptor is required for response to the sugar trehalose in taste neurons of Drosophila. Nat Neurosci 4:1182-1186.

Dunipace L, Meister S, McNealy C, Amrein H (2001) Spatially restricted expression of candidate taste receptors in the Drosophila gustatory system. Curr Biol 11:822-835.

Falk R, Bleiser-Avivi N, Atidia J (1976) Labellar taste organs of Drosophila melanogaster. J Morph 150:327-342.

Fujishiro N, Kijima H, Morita H (1984) Impulse frequency and action potential amplitude in labellar chemosensory neurons of Drosophila melanogaster. J Insect Physiol 30:317-325.

Hiroi M, Marion-Poll F, Tanimura T (2002) Differentiated response to sugars among labellar chemosensilla in Drosophila. Zoolog Sci 19:1009-1018.

Hiroi M, Meunier N, Marion-Poll F, Tanimura T (2004) Two antagonistic gustatory receptor neurons responding to sweet-salty and bitter taste in Drosophila. J Neurobiol 61:333-342.

Hodgson ES, Lettvin JY, Roeder KD (1955) Physiology of a primary chemoreceptor unit. Science 122:417-418.

Hou D, Suzuki K, Wolfgang WJ, Clay C, Forte M, Kidokoro Y (2003) Presynaptic impairment of synaptic transmission in Drosophila embryos lacking Gs $\alpha$. J Neurosci 23:5897-5905.

Hurley JH (1999) Structure, mechanism, and regulation of mammalian adenylyl cyclase. J Biol Chem 274:7599-7602.

Ishimoto H, Takahashi K, Ueda R, Tanimura T (2005) G-protein gamma subunit 1 is required for sugar reception in Drosophila. EMBO J 24:3259-3265.

Jones DT, Reed RR (1989) Golf: an olfactory neuron specific-G protein involved in odorant signal transduction. Science 244:790-795.

Koganezawa M, Shimada I (2002) Inositol 1,4,5-trisphosphate transduction cascade in taste reception of the fleshfly, Boettcherisca peregrina. J Neurobiol 51:66-83.

Liu L, Leonard AS, Motto DG, Feller MA, Price MP, Johnson WA, Welsh MJ (2003) Contribution of Drosophila DEG/ENaC genes to salt taste. Neuron 39:133-146.

Luo L, Liao YJ, Jan LY, Jan YN (1994) Distinct morphogenetic functions of similar small GTPases: Drosophila Dracl is involved in axonal outgrowth and myoblast fusion. Genes Dev 8:1787-1802.

Menco BP, Bruch RC, Dau B, Danho W (1992) Ultrastructural localization 
of olfactory transduction components: the G protein subunit Golf alpha and type III adenylyl cyclase. Neuron 8:441-453.

Meunier N, Marion-Poll F, Rospars JP, Tanimura T (2003) Peripheral coding of bitter taste in Drosophila. J Neurobiol 56:139-152.

Murata Y, Mashiko M, Ozaki M, Amakawa T, Nakamura T (2004) Intrinsic nitric oxide regulates the taste response of the sugar receptor cell in the blowfly, Phormia regina. Chem Senses 29:75-81.

Naim M, Ronen T, Striem BJ, Levinson M, Zehavi U (1991) Adenylate cyclase responses to sucrose stimulation in membranes of pig circumvallate taste papillae. Comp Biochem Physiol B 100:455-458.

Neves SR, Ram PT, Iyengar R (2002) G protein pathways. Science 296:1636-1639.

Pearn MT, Randall LL, Shortridge RD, Burg MG, Pak WL (1996) Molecular, biochemical, and electrophysiological characterization of Drosophila norpA mutants. J Biol Chem 271:4937-4945.

Quan F, Forte MA (1990) Two forms of Drosophila melanogaster Gs $\alpha$ are produced by alternate splicing involving an unusual splice site. Mol Cell Biol 10:910-917.

Quan F, Wolfgang WJ, Forte MA (1989) The Drosophila gene coding for the $\alpha$ subunit of a stimulatory $\mathrm{G}$ protein is preferentially expressed in the nervous system. Proc Natl Acad Sci USA 86:4321-4325.

Robertson HM, Warr CG, Carlson JR (2003) Molecular evolution of the insect chemoreceptor gene superfamily in Drosophila melanogaster. Proc Natl Acad Sci USA 100 [Suppl 2]:14537-14542.

Scott K, Brady Jr R, Cravchik A, Morozov P, Rzhetsky A, Zuker C, Axel R (2001) A chemosensory gene family encoding candidate gustatory and olfactory receptors in Drosophila. Cell 104:661-673.

Shimada I, Nakao M, Kawazoe Y (1987) Acute differential sensitivity and role of the central nervous system in the feeding behavior of Drosophila melanogaster. Chem Senses 12:481-490.

Simonds WF (1999) G protein regulation of adenylate cyclase. Trends Pharmacol Sci 20:66-73.
Striem BJ, Pace U, Zehavi U, Naim M, Lancet D (1989) Sweet tastants stimulate adenylate cyclase coupled to GTP-binding protein in rat tongue membranes. Biochem J 260:121-126.

Talluri S, Bhatt A, Smith DP (1995) Identification of a Drosophila G protein alpha subunit ( $\mathrm{dGq} \alpha-3)$ expressed in chemosensory cells and central neurons. Proc Natl Acad Sci USA 92:11475-11479.

Tesmer JJ, Sprang SR (1998) The structure, catalytic mechanism and regulation of adenylyl cyclase. Curr Opin Struct Biol 8:713-719.

Thorne N, Chromey C, Bray S, Amrein H (2004) Taste perception and coding in Drosophila. Curr Biol 14:1065-1079.

Ueno K, Ohta M, Morita H, Mikuni Y, Nakajima S, Yamamoto K, Isono K (2001) Trehalose sensitivity in Drosophila correlates with mutations in and expression of the gustatory receptor gene Gr5a. Curr Biol 11:1451-1455.

Wang Z, Singhvi A, Kong P, Scott K (2004) Taste representations in the Drosophila brain. Cell 117:981-991.

Wolfgang WJ, Quan F, Goldsmith P, Unson C, Spiegel A, Forte M (1990) Immunolocalization of $\mathrm{G}$ protein alpha-subunits in the Drosophila CNS. J Neurosci 10:1014-1024.

Wolfgang WJ, Hoskote A, Roberts IJ, Jackson S, Forte M (2001) Genetic analysis of the Drosophila Gs $\alpha$ gene. Genetics 158:1189-1201.

Wolfgang WJ, Clay C, Parker J, Delgado R, Labarca P, Kidokoro Y, Forte M (2004) Signaling through Gs $\alpha$ is required for the growth and function of neuromuscular synapses in Drosophila. Dev Biol 268:295-311.

Wong SK (2003) G protein selectivity is regulated by multiple intracellular regions of GPCRs. Neurosignals 12:1-12.

Zhang Y, Hoon MA, Chandrashekar J, Mueller KL, Cook B, Wu D, Zuker CS, Ryba NJ (2003) Coding of sweet, bitter, and umami tastes: different receptor cells sharing similar signaling pathways. Cell 112:293-301.

Zhu L, McKay RR, Shortridge RD (1993) Tissue-specific expression of phospholipase $\mathrm{C}$ encoded by the norpA gene of Drosophila melanogaster. J Biol Chem 268:15994-16001. 